

\title{
Investigation of $\pi^{+} \pi^{-}$and $\pi K$ atoms at DIRAC
}

\author{
Valeriy Yazkov ${ }^{* \dagger}$ \\ SINP MSU \\ E-mail: Valeri.Yazkovecern.ch
}

\begin{abstract}
Measurement of $\pi^{+} \pi^{-}$and $\pi K$ atom lifetimes allows to check prediction of Chiral Perturbation Theory for pion-pion scattering lengths with isospin 0 and 2, and pion-kaon scattering lengths with isospin $1 / 2$ and 3/2. Current status of DIRAC experiment, expected results and proposal for future investigations are presented.
\end{abstract}

6th International Workshop on Chiral Dynamics

July 6-10 2009

Bern, Switzerland

\footnotetext{
*Speaker.

${ }^{\dagger}$ on behalf of DIRAC Collaboration
} 


\section{Introduction}

Pionium $\left(A_{2 \pi}\right)$ is a hydrogen-like atom consisting of $\pi^{+}$and $\pi^{-}$mesons:

$$
E_{B}=-1.86 \mathrm{keV}, r_{B}=387 f m, p_{B} \approx 0.5 \mathrm{MeV} / \mathrm{c} .
$$

Here $E_{B}$ is bounding energy, $r_{B}$ and $p_{B}$ are Bohr radius and Bohr momentum, correspondingly. The $\pi^{+} \pi^{-}$atom decays by strong interaction mainly into $\pi^{0} \pi^{0}$. The branching ratio of the alternative decay mode $A_{2 \pi} \rightarrow 2 \gamma$ is at the level of $4 \cdot 10^{-3}$. There is a relation [1] between the width of $A_{2 \pi}$ decay $\Gamma_{1 s, 2 \pi^{0}}$ and $\pi \pi$ scattering lengths for isospin 0 and 2:

$$
\Gamma_{1 s, 2 \pi^{0}}=R \cdot\left|a_{0}-a_{2}\right|^{2} .
$$

The scattering lengths $a_{0}$ and $a_{2}$ have been calculated in Chiral Perturbation Theory (ChPT) with a precision better than $2.5 \%$ [B] : $a_{0}=0.220 \pm 0.005, a_{2}=-0.0444 \pm 0.0010$ and $a_{0}-a_{2}=$ $0.265 \pm 0.004$.

Precision of these results depends on the low-energy constants (LEC) $l_{3}$ and $l_{4}$. Because $l_{3}$ and $l_{4}$ are sensitive to the quark condensate, precise measurements of $a_{0}, a_{2}$ are a way to study the structure of the QCD vacuum.

ChPT at next-to-leading order in isospin breaking provides coefficient $R$ at Eq. 1.1 is known with accuracy $\Delta R / R=1.2 \%$ [ [. It allows to predict $A_{2 \pi}$ lifetime to be:

$$
\tau=(2.9 \pm 0.1) \times 10^{-15} s
$$

The goal of the DIRAC experiment at CERN (PS212) at the first stage was to measure the pionium lifetime with $10 \%$ precision. Such a measurement provides in a model independent way the difference between the S-wave $\pi \pi$ scattering $\left|a_{0}-a_{2}\right|$, with 5\% accuracy. Therefore, such a measurement will be a sensitive check of ChPT prediction and understanding of chiral symmetry breaking in QCD.

At the second stage of DIRAC experiment [F] observation and the first experimental measurement of $\pi K$ atoms are expected, and accuracy of $A_{2 \pi}$ lifetime measurement is to be improved up to $\sigma_{\tau} / \tau=4 \%$ which provides measurement of pionium scattering length difference with accuracy $\sigma_{\left|a_{0}-a_{2}\right|}|| a_{0}-a_{2} \mid=2 \%$.

$\pi K$-atom $\left(A_{\pi K}\right)$ is a hydrogen-like atom consisting of $K^{+}\left(K^{-}\right)$and $\pi^{-}\left(\pi^{+}\right)$mesons. Its features are:

$$
E_{B}=-2.9 \mathrm{keV}, r_{B}=248 \mathrm{fm}, p_{B}=0.8 \mathrm{MeV} / \mathrm{c} .
$$

The $\mathrm{K}$-atom lifetime (ground state $1 \mathrm{~S}$ ), $\tau=1 / \Gamma$ is defined by the annihilation process into $K^{0} \pi^{0}$ :

$$
A_{K^{+} \pi^{-}} \rightarrow \pi^{0} K^{0}, A_{\pi^{+} K^{-}} \rightarrow \pi^{0} \bar{K}^{0}
$$

Width of $A_{\pi K}$ decay $\Gamma_{1 s, K^{0} \pi^{0}}$ is proportional to squared difference of $\pi K$ scattering lengths for isospin $1 / 2$ and $3 / 2$ [6]: 


$$
\Gamma_{1 s, K^{0} \pi^{0}}=R_{K}\left|a_{1 / 2}-a_{3 / 2}\right|^{2}, \frac{\sigma_{R_{K}}}{R_{K}}=2 \% .
$$

At present there are two predictions for pion-kaon scattering lengths. ChPT predicts s-wave scattering lengths to be [7], [8]:

$$
a_{1 / 2}=0.19 \pm 0.2, a_{3 / 2}=-0.05 \pm 0.02, a_{1 / 2}-a_{3 / 2}=0.23 \pm 0.01 .
$$

Another prediction for scattering length difference have been obtained in [9] using Roy-Steiner equations:

$$
a_{1 / 2}-a_{3 / 2}=0.269 \pm 0.015
$$

With prediction from Eq. 1.4 lifetime of $A_{\pi K}$ in ground state estimated to be:

$$
\tau=(3.7 \pm 0.4) \times 10^{-15}
$$

The measurement of the s-wave $\mathrm{K}$ scattering lengths would test our understanding of the chiral $S U(3)_{L} \times S U(3)_{R}$ symmetry breaking of QCD (u, d and s quarks), while the measurement of $\pi \pi$ scattering lengths checks only the $S U(2)_{L} \times S U(2)_{R}$ symmetry breaking (u, d quarks). This is the principal difference between $\pi \pi$ and $\pi K$ scattering. Experimental data on the $\mathrm{K}$ low-energy phases are absent now.

\section{Method of lifetime measurement}

The $A_{2 \pi}$ are produced by Coulomb interaction in the final state of $\pi^{+} \pi^{-}$pairs generated in proton-target interactions from fragmentation and strong decay ("short-lived" sources). For this cases the region of production being small as compared to the Bohr radius of the atom [10].

Also $\pi^{+} \pi^{-}$pairs from short-lived sources are generated in free state. Such pairs ("Coulomb pairs") are affected by Coulomb interaction, too. The number of produced atoms $\left(N_{A}\right)$ is proportional to the number of "Coulomb pairs" $\left(N^{C}\right)$ with low relative momentum $\left(N_{A}=K \cdot N^{C}\right)$. The coefficient $K$ is precisely calculable. And there are $\pi^{+} \pi^{-}$pairs from long-lived sources (electromagnetically or weakly decaying mesons or baryons: $\left.\eta, K_{s}^{0}, \ldots\right)$. Such pairs, not affected by final state interaction, are named "non-Coulomb pairs".

Another type of background is "accidental pairs" consisted of pions generated in two different proton-nucleus. They are also not affected by final state interaction.

After production $A_{2 \pi}$ travel through the target and some of them are broken up due to their interaction with matter: "atomic pairs" are produced, characterized by small pair c.m. relative momentum $Q<3 \mathrm{MeV} / c$. These pairs are detected by the DIRAC setup. Other atoms annihilate into $\pi^{0} \pi^{0}$. Using experimentally measured number of "Coulomb" pairs it is possible to measure breakup probability $P_{\mathrm{br}}(\tau)=n_{A} / N_{A}=n_{A} /\left(K \cdot N_{C}\right)$.

The dependence of $P_{\mathrm{br}}$ on the lifetime $\tau$ is determined by the solution of differential transport equations [11]. In fig. 1 the lifetime dependence of $P_{\mathrm{br}}$ is presented for three different targets used in the DIRAC experiment. The nickel target provides the best statistical accuracy for the same running time.

The same method is applied to $\pi K$ atoms. 


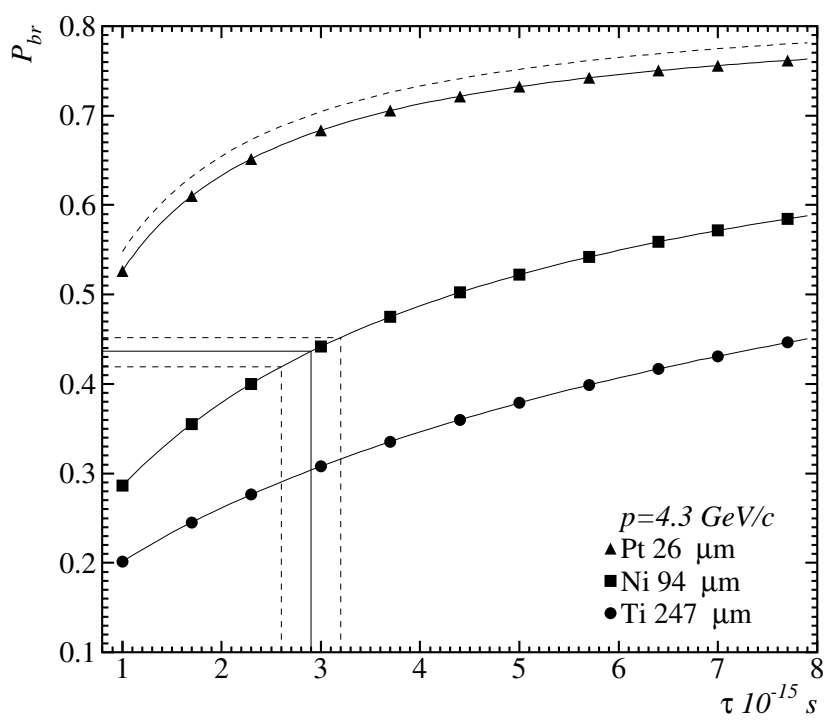

Figure 1: Dependence of the breakup probability $P_{\mathrm{br}}$ on $A_{2 \pi}$ lifetime for three targets used in the DIRAC experiment: platinum of $26 \mu \mathrm{m}$, nickel of $94 \mu \mathrm{m}$ and titanium of $247 \mu \mathrm{m}$ thickness.

\section{Experimental setup}

DIRAC setup was created to detect $\pi^{+} \pi^{-}$with small relative momenta [12]. In 2004-2006 it has been modified in order to detect both $\pi^{+} \pi^{-}$and $\pi K$ pairs from broken atoms. New detector for particle identification have been added: Cherenkov counters with heavy gas and Aerogel. Taking into account kinematic of $\pi K$ "atomic pairs", new detectors cover only internal parts of each arm (see Fig. 2).

\section{Analysis}

Analysis is based on description of experimental data with simulated distribution of "atomic pairs" and background pairs: "Coulomb pairs" and "non-Coulomb pairs".

Atoms are generated in $n s$ states using measured momentum distribution of $\pi^{+} \pi^{-}$pairs for short-lived sources. The atomic pairs are generated according to the evolution of the atom while propagating through the target (see section 2).

Coulomb pairs are generated according to $A_{C}(Q) \cdot Q^{2}$ in pair c.m. system. Here $A_{C}(Q)$ is Gamow-Sommerfeld factor and $Q^{2}$ is a phase space. Distribution over total pair momentum in Lab system is simulated in accordance to measured momentum distribution of $\pi^{+} \pi^{-}$for short-lived sources as for the case of atoms.

Non-Coulomb pairs are generated according to $Q^{2}$ in pair c.m. using measured total momentum distribution of $\pi^{+} \pi^{-}$for long-lived sources.

Experimental data are fit with a sum of "atomic", "Coulomb" and "non-Coulomb" pair distributions. Weight coefficient are free parameters of fit. It allows to obtain both "atomic pair" number $n_{A}$ and number of generated atoms $N_{A}$ with a measured number of "Coulomb pairs". 


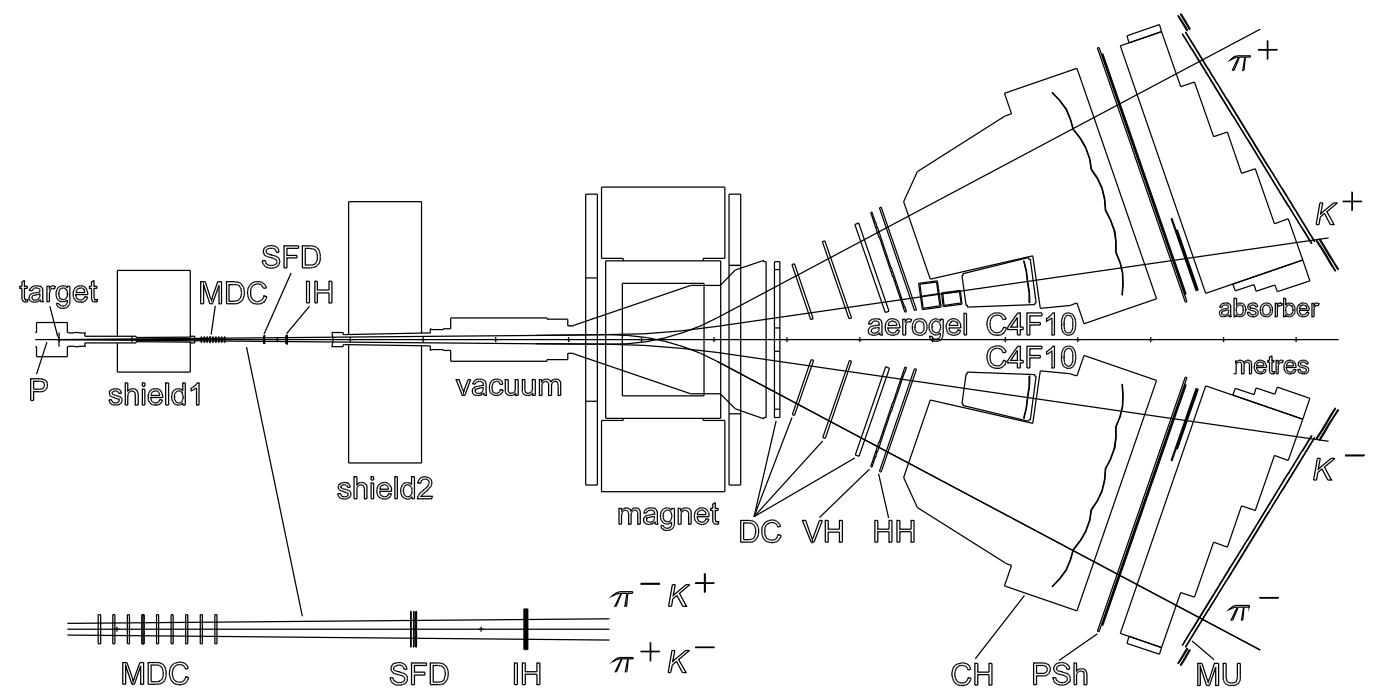

Figure 2: Updated DIRAC setup: MDC are microdrift gas chambers, SFD is a scintillating fiber detector and $\mathrm{IH}$ is a scintillation ionization hodoscope. Downstream the spectrometer magnet there are drift chambers (DC), vertical ( $\mathrm{VH})$ and horizontal (HH) scintillation hodoscopes. Cherenkov detectors contain nitrogen (CH), heavy gas C4F10 and aerogel radiators. Shower detectors (PSh) and scintillation muon detectors (MU).

\section{DIRAC Experimental results}

The first DIRAC result was based on statistic collected in 2001 (6530 observed $\pi^{+} \pi^{-}$"atomic pairs"). It allows to obtain lifetime measurement with total error $\sim 18 \%$ [13]:

$$
\tau=\left(\left.\left.2.91_{-0.38}^{+0.45}\right|_{\text {stat }}{ }_{-0.49}^{+0.19}\right|_{\text {syst }}\right) f s=\left(\left.\ldots{ }_{-0.62}^{+0.49}\right|_{\text {tot }}\right) .
$$

It follows with a measurement of pion-pion scattering length difference:

$$
\left|a_{0}-a_{2}\right|=0.264 \pm\left. 7.2 \%{ }_{\text {stat }}{ }_{-3}^{+10} \%\right|_{\text {syst }}=\left.\ldots{ }_{-8}^{+13} \%\right|_{\text {tot }} .
$$

At present all data collected in 2001-2003 have been analyzed and new estimation of lifetime is prepared on the base of 13300 "atomic pairs":

$$
\begin{aligned}
\tau & =\left(\left.2.82_{-0.23}^{+0.25}\right|_{\text {stat }} \pm 0.19_{\text {syst }}\right) f s=\left(\left.\ldots{ }_{-0.30}^{+0.31}\right|_{\text {tot }}\right) \\
\left|a_{0}-a_{2}\right| & =0.268 \pm 4.4 \%_{\text {stat }} \pm 3.7 \%_{\text {syst }}=\ldots \pm 5.5 \%_{\text {tot }}
\end{aligned}
$$

These results are not final for data collected in 2001-2003 because they have been obtained without using of data from MSGC. This detector has high resolution and efficiency. It open possibility to increase statistic of "atomic pairs" at least up to 17000 and to provide a statistical error in $\left|a_{0}-a_{2}\right|$ at the level 3\%, and an expected total error is better than 5\%.

Analysis of data collected in 2008 with platinum target allows to make estimation of experimental conditions for observation of $\pi K$ atoms and their lifetime measurements [14]. 


\section{Comparison with other experimental results}

Among other experimental ways for measurement of pion-pion scattering lengths it is needed to mention experiment with $K_{e 4}\left(K \rightarrow \pi^{+} \pi^{-} e v_{e}\right)$. The final state interaction can be studied by analyzing the difference between the s- and p-wave scattering phases. It provides model-independent measurement of scattering length. The latest result obtained by NA48/2 experiment are presented in [15] with free $a_{2}$ :

$$
\begin{aligned}
& a_{0}=0.233 \pm 6.9 \%_{\text {stat }} \pm 3.0 \%_{\text {syst }}=\ldots \pm 7.5 \%_{\text {tot }}, \\
& a_{2}=-0.0471 \pm 23 \%_{\text {stat }} \pm 8.5 \%_{\text {syst }}=\ldots \pm 25 \%_{\text {tot }} .
\end{aligned}
$$

In 2009 updated version of these results has been presented at CERN seminar with free $a_{2}$ :

$$
\begin{aligned}
& a 0=0.2220 \pm 5.8 \%_{\text {stat }} \pm 2.3 \%_{\text {syst }} \pm 1.7 \%_{\text {th }}=\ldots \pm 6.5 \%_{\text {tot }}, \\
& a 2=-0.0432 \pm 20 \%_{\text {stat }} \pm 7.9 \%_{\text {syst }} \pm 6.5 \%_{\text {th }}=\ldots \pm 22 \%_{\text {tot }},
\end{aligned}
$$

and with ChPT constraint between $a_{0}$ and $a_{2}$ :

$$
a 0=0.2206 \pm 2.2 \%_{\text {stat }} \pm 0.8 \%_{\text {syst }} \pm 2.9 \%_{\text {th }}=\ldots \pm 3.7 \%_{\text {tot }} .
$$

Another method to measure difference of pion-pion scattering lengths is "cusp-effect" in $K \rightarrow$ $3 \pi$. NA48/2 collaboration has presented results [16] using ChPT constrains between $a_{0}$ and $a_{2}$ :

$$
a_{0}-a_{2}=0.264 \pm 2.3 \%_{\text {stat }} \pm 1.5 \%_{\text {syst }} \pm 4.9 \%_{\text {ext }}=\ldots \pm 5.6 \%_{\text {tot }} .
$$

In 2009 updated version of these results has been presented at CERN seminar with free $a_{2}$ :

$$
\begin{aligned}
a_{0}-a_{2} & =0.257 \pm 1.9 \%_{\text {stat }} \pm 0.8 \%_{\text {syst }} \pm 0.4 \%_{\text {ext }} \pm 3.5 \%_{\text {th }}=\ldots \pm 4.1 \%_{\text {tot }}, \\
a_{2} & =-0.024 \pm 54 \%_{\text {stat }} \pm 38 \%_{\text {syst }} \pm 8.3 \%_{\text {ext }} \pm 63 \%_{\text {th }}=\ldots \pm 92 \%_{\text {tot }}
\end{aligned}
$$

and with ChPT constraint between $a_{0}$ and $a_{2}$ :

$$
a_{0}-a_{2}=0.263 \pm 0.8 \%_{\text {stat }} \pm 0.4 \%_{\text {syst }} \pm 0.8 \%_{\text {ext }} \pm 1.9 \%_{\text {th }}=\ldots \pm 2.2 \%_{\text {tot }} .
$$

It is seen that present experiments provide measurement of pion-pion scattering length with compatible accuracy but in different approaches. This increases reliability of ChPT prediction check.

\section{Future investigations at DIRAC}

It is proposed to perform measurement of energy splitting between $n p$ and $n s$ states of $\pi^{+} \pi^{-}$ atoms. The energy shift for the levels with the principal quantum number $n$ and orbital quantum number $l$ includes few contributions [6]: 


\begin{tabular}{|l|l|l|l|l|}
\hline$n$ & $l$ & $\Delta E_{n l}^{e m}$ & $\Delta E_{n l}^{v a c}$ & $\Delta E_{n l}^{s t r}$ \\
\hline 1 & 0 & -0.065 & -0.942 & $-3.80 \pm 0.1$ \\
2 & 0 & -0.012 & -0.111 & $-0.47 \pm 0.01$ \\
2 & 1 & -0.004 & -0.004 & $-1 \times 10^{-6}$ \\
\hline
\end{tabular}

Table 1: Numerical values for the energy shift.

$$
\Delta E_{n l}=\Delta E_{n l}^{e m}+\Delta E_{n l}^{v a c}+\Delta E_{n l}^{s t r},
$$

where $\Delta E_{n l}^{e m}$ takes into account finite-size effect, relativistic correction and corrections due to transverse photons and transverse photon exchange. The term $\Delta E_{n l}^{v a c}$ is the contribution from the vacuum polarization. The last term $\Delta E_{n l}^{s t r}$ takes into account strong interaction effects and is related to the scattering lengths as follows:

$$
\Delta E_{n 0}^{s t r}=A_{n}\left(2 a_{0}+a_{2}\right) .
$$

And the theoretical value for the $2 \mathrm{~s}-2 \mathrm{p}$ energy splitting is:

$$
\Delta E^{2 p-2 s}=\Delta E_{20}^{s t r}+\Delta E_{20}^{e m}-\Delta E_{21}^{e m}+\Delta E_{20}^{v a c}-\Delta E_{21}^{v a c}=-0.59 \pm 0.01 \mathrm{eV}
$$

Prediction for energy shift values [6] are presented in Tab. 1 for different $n l$-states.

By measuring the value of $\Delta E^{n p-n s}$ one can determine the numerical value of $\Delta E_{20}^{s t r}$, as all other terms in Eq. 7.1 have been calculated with a high accuracy. From Eq. 7.3 and Table 1 it follows that the strong interaction effects contributes up to $80 \%$ of the full energy shift. This fact provides a high sensitivity of a $\Delta E^{2 p-2 s}$ measurement to the value of the term $\left|2 a_{0}+a_{2}\right|$. Thus, measurements of the energy shift $\Delta E_{n}$ make it possible to obtain values for the new combination of scattering lengths $\left|2 a_{0}+a_{2}\right|$ in a model-independent way.

Measurement of energy shift $\Delta E_{n}$ is possible with metastable $\pi^{+} \pi^{-}$atoms. Lifetime $\tau_{n p}$ and length of path before annihilation $\lambda_{n p}$ for $A_{2 \pi}$ in $p$-state are much more than for $s$-state with the same principal quantum number:

$$
\lambda_{1 s}=1.7 \times 10^{-3} \mathrm{~cm}, \lambda_{2 s}=1.4 \times 10^{-2} \mathrm{~cm}, \lambda_{2 p}=7 \mathrm{~cm}, \lambda_{3 p}=23 \mathrm{~cm}, \lambda_{4 p}=54 \mathrm{~cm},
$$

In inclusive processes $A_{2 \pi}$ are produced in s-states distributed over the principal quantum number $n$ proportionally to $n^{-3}$. When moving inside the target, the relativistic $A_{2 \pi}$ interacts with the electric field of the target atoms and with some probability will leave the target with orbital momentum $l \neq 0$. Calculations show that up to $\sim 10 \%$ of the atoms, generated in a thin target, reach the vacuum region in a long-lived state [17]. The main part of these atoms will be in the $2 p$-state (see Table 2):

Metastable atoms could be observed using two layer target. One thin layer is in the proton beam. It produces "atomic pairs" from break up and exited atoms which go to vacuum after the first layer. Atoms in $s$-states annihilate but atoms with $l \geq 1$ could achieve the second layer and to be broken there. 


\begin{tabular}{|r|c|c|c|c|c|c|c|}
\hline $\begin{array}{c}\text { Target } \\
\mathrm{Z}\end{array}$ & $\begin{array}{c}\text { Thickness } \\
\mu\end{array}$ & $\begin{array}{c}P_{B r} \\
\%\end{array}$ & $\begin{array}{c}\sum \\
(l \geq 1)\end{array}$ & $\begin{array}{c}2 \mathrm{p} 0 \\
\%\end{array}$ & $\begin{array}{c}3 \mathrm{p} 0 \\
\%\end{array}$ & $\begin{array}{c}4 \mathrm{p} 0 \\
\%\end{array}$ & $\begin{array}{c}\sum \\
(l=1, m=0)\end{array}$ \\
\hline 4 & 100 & 4.45 & 5.86 & 1.05 & 0.46 & 0.15 & 1.90 \\
6 & 50 & 5.00 & 6.92 & 1.46 & 0.51 & 0.16 & 2.52 \\
13 & 20 & 5.28 & 7.84 & 1.75 & 0.57 & 0.18 & 2.63 \\
28 & 5 & 9.42 & 9.69 & 2.40 & 0.58 & 0.18 & 3.29 \\
78 & 2 & 18.8 & 10.5 & 2.70 & 0.54 & 0.16 & 3.53 \\
\hline
\end{tabular}

Table 2: Probabilities of $A_{2 \pi}$ breakup $\left(P_{B r}\right)$ and yields of the long-lived states for different targets, provided the maximum yield of summed population of the long-lived states: $\Sigma(l \geq 1)$.

The transverse magnetic field $B_{0}$ in the lab system is increased in the atom reference system as $B=\gamma B_{0}$ ( $\gamma$ is Lorentz factor). The corresponding electric field has nearly the same amplitude $F=\beta B$ ( $\beta$ is the atom velocity in the lab frame) and is perpendicular to the atom momentum.

In electric fields $p$-states are mixed with $s$-states and it causes significant acceleration of the annihilation. As result fraction of atoms which destine the second layer would decreases dependently on magnetic field and energy shift values. It allows to measure energy shift and $\pi \pi$ scattering length combination $\left|2 a_{0}+a_{2}\right|$.

\section{Prospect for creation of intense source of exotic atoms}

Creation of an intense source of $\pi^{+} \pi^{-}, \pi K$ and other exotic atoms at SPS proton beam allows to perform accurate measurements of all S-wave $\pi \pi$ and $\pi K$ scattering length to check the precise low energy QCD prediction.

Table 3 shows that investigation of $\pi \pi$ and $\pi K$ atoms at SPS energy provide essential gain in statistic and hence in accuracy. Increasing of proton beam momentum leads to growth of yield both atoms $\left(W_{A}\right)$ and single charged particles $\left(W_{\pi}\right)$. Flux of single particles limits maximal beam intensity and possibility to increase atom production rate with intensity. But growth of atom yield is high. Therefore for the same flux of single charged particle number of produced atoms increases as $W_{A} / W_{\pi}$. Additional gain is provided by better beam structure of SPS. Totally source of exotic atoms on SPS allows to obtain needed accuracy faster in $15 \div 30$ times.

\section{Summary}

In close future DIRAC will present $\pi^{+} \pi^{-}$atom lifetime on the base of data collected in 20012003. It allows to achieve claimed accuracy of $10 \%$ for lifetime and 5\% for $\left|a_{0}-a_{2}\right|$.

Data of second stage of experiment in 2007-2009 (2010?) give possibility to double statistic for $\pi^{+} \pi^{-}$atoms, to make observation of $\pi K$-atom and to estimate its lifetime.

DIRAC plans foreseen further development of investigation of $A_{2 \pi}, A_{\pi K}$ and possibly other exotic atoms on order to check predictions of ChPT for $\pi \pi$ and $\pi K$ scattering lengths.

\section{References}

[1] J. Uretsky and J. Palfrey, Phys. Rev., 121 (1961) 1798. 


\begin{tabular}{|l|c|c|c|c|c|}
\hline Atom & $W_{A}$ & $W_{A}^{N}$ & $\frac{W_{A}}{W_{\pi}}$ & $\left.\frac{W_{A}}{W_{\pi}}\right|^{N}$ & $\begin{array}{c}\text { total } \\
\text { gain }\end{array}$ \\
\hline \multicolumn{6}{|c|}{$24 \mathrm{GeV}$} \\
\hline$A_{\pi^{+} \pi^{-}}$ & $1.10 \cdot 10^{-9}$ & 1. & $3.4 \cdot 10^{-8}$ & 1. & 1. \\
$A_{K^{+} \pi^{-}}$ & $0.52 \cdot 10^{-10}$ & 1. & $16.10^{-10}$ & 1. & 1. \\
$A_{\pi^{+} K^{-}}$ & $0.29 \cdot 10^{-10}$ & 1. & $9.0 \cdot 10^{-10}$ & 1. & 1. \\
\hline \multicolumn{6}{|c|}{$450 \mathrm{GeV}$} \\
\hline$A_{\pi^{+} \pi^{-}}$ & $0.13 \cdot 10^{-7}$ & 12. & $1.3 \cdot 10^{-7}$ & 3.8 & 15. \\
$A_{K^{+} \pi^{-}}$ & $0.10 \cdot 10^{-8}$ & 19. & $1.0 \cdot 10^{-8}$ & 6.2 & 25. \\
$A_{\pi^{+} K^{-}}$ & $0.71 \cdot 10^{-9}$ & 24. & $7.1 \cdot 10^{-9}$ & 8.0 & 32. \\
\hline
\end{tabular}

Table 3: Yield of dimeson atoms per one proton-Ni interaction $\left(W_{A}\right)$, detectable by DIRAC upgrade setup at $\Theta_{L}=5.7^{\circ}$; yield of atoms normalized on yield at proton beam $24 \mathrm{GeV} / c\left(W_{A}^{N}\right)$; ratio of $W_{A}$ to inclusive cross-section of charged particles $W_{\pi}$; normalized ratio $\left.\frac{W_{A}}{W_{\pi}}\right|^{N}$; total gain with SPS beam relatively PS beam, taking in to account spill frequency and duration.

[2] S.M. Bilenky et al., Sov. J. Nucl. Phys. 10 (1969) 469.

[3] G. Colangelo et al., Nucl. Phys. B 603 (2001) 125.

[4] J. Gasser et al., Phys. Rev. D64 (2001) 016008 [hep-ph/ 0103157$].$

[5] B. Adeva et al., LIFETIME MEASUREMENT OF $\pi^{+} \pi^{-}$AND $\pi^{ \pm} K^{\mp}$ ATOMS TO TEST LOW ENERGY QCD, CERN-SPSC-2004-009, SPSC-P-284 Add.4, CERN 2004.

[6] J. Schweizer, Phys. Lett. B 587 (2004) 33 [arXiv: hep-ph / 0401048 ].

[7] V. Bernard, N. Kaiser and U. Meissner, Phys. Rev. D43 (1991) 2757.

[8] A. Roessl, Nucl. Phys B555 (1999) 507 [hep-ph/ 9904230$].$

[9] P. Buttiker, S. Descotes-Genon and B. Moussallam, (2004) [hep-ph/0310283].

[10] L. Nemenov, Sov. J. Nucl. Phys. 41 (1985) 629.

[11] L. Afanasyev and A. Tarasov, Phys. At. Nucl. 59 (1996) 2130.

[12] B. Adeva et al., Nucl. Instrum. Methods A 515 (2003) 467.

[13] B. Adeva et al., Phys. Lett. B 619 (2005) 50.

[14] B. Adeva et al., Phys. Lett. B 674 (2009) 11.

[15] J. Batley et al., Eur. Phys. J. C54 (2008) 411.

[16] J. Batley et al., Phys. Lett. B 633 (2006) 173 [hep-ex/ 0511056 ].

[17] L. Afanasyev, Observation of $\pi^{+} \pi^{-}$atom, PhD thesis, JINR Dubna 1997. 